\title{
Dynamics of supermassive black holes
}

\author{
Marc Hemsendorf, Nils Dorband and David Merritt \\ Department of Physics and Astronomy, Rutgers, the State University of \\ New Jersey, 136 Frelinghuysen Road, Piscataway, NJ 08854-8019, USA
}

\begin{abstract}
We are showing first results from simulations studying the effect of Brownian motion of the central mass on a cusp. N-body simulations of cuspy stellar systems around supermassive black holes represent a very challenging computational task. The wide range of dynamical timescales combined with large particle numbers required here leads us to the implementation of a systolic force calculation into a Hermite direct force block time-step scheme. With this new algorithm, we are able to handle particle numbers as large as 900000 on the parallel computers accessible to us. Due to their extraordinary parallelism (up to $98 \%$ and more) we suggest to use the described schemes on a computer cluster where each computing node is connected to a GRAPE board.
\end{abstract}

\section{Method}

We call our approach a systolic algorithm, because phases of parallel work are followed by communiation as it could be visualized by the blood circulation in an organism. This term has been coined by Kung \& Leiserson (1978). Our systolic code implements a circular routing scheme which is responsible for summation partial forces computed by each node. The underlying integrator follows the Hermite scheme with individual block time-steps (Makino \& Aarseth 1992). Our systolic scheme allows for optimal data distribution on distributed memory parallel architectures (e.g. computer clusters, parallel supercomputers etc.) The data transfer to the neighboring processors is delegated to a subthread so that the processing elements carry out their computations while communicating.

\section{Brownian motion of a massive particle}

With the density and velocity dispersion given from the $\gamma=2$ Dehnen model, we can estimate the timescale for equipartition between our black hole particle $\left(m_{\bullet}=0.001,0.01\right.$, and 0.1$)$ and the rest of our model $\left(m_{*}=0.000002, N=\right.$ $500000)$.

$$
\begin{aligned}
& T_{\mathrm{eq}} \approx \frac{3 \sqrt{\pi}}{4 \ln (\Lambda)}\left(\frac{m_{\bullet}}{M}\right) \sqrt{\frac{a^{3}}{G M}}, \\
& T_{\mathrm{eq}} \approx 10^{-3} T_{d} .
\end{aligned}
$$


For our simulation we have $a=1, M=1, G=1$. Please note: We do not apply any softening! This estimation is reasonably shorter than the value which one can derive from the classical Spitzer equipartition timescale which would yield $T_{\text {spitzer }} \approx 10 T_{d}$ (Spitzer 1987 ) for a black hole of mass $m_{\bullet}=0.001$ in our system. In our simulations we find that the massive particle adjusts to its expected equipartition velocity within a very short timescale, which agrees with our estimate above.

\section{Summary}

Systolic schemes provide a simple and effiecient way to port individual time-step codes to distributed memory machines. Hypersystolic methods can tune such codes for smaller problem sizes or larger computing node numbers. However they are harder to implement and provide not such as perfect loadbalancing as systolic schemes. We are able to integrate up to 900000 particles on 128 nodes of a Cray T3E 900 supercomputer with a crude individual block time-step Hermite direct force method for several dynamical timescales. With our simulations we can show that equipartition between a massive black hole and the surrounding stars can be reached in a very short timescale.

\section{References}

Kung, H. T. \& Leiserson, C. E. 1978, in Sparse matrix proceedings, ed. Duff, I. S. \& Stewart, G. W. (Philadelphia: SIAM), 256

Makino, J. \& Aarseth, S. J. 1992, PASJ, 44, 141

Spitzer, L. 1987, Dynamical evolution of globular clusters (Princeton: Princeton University Press)

\section{Acknowledgements}

The authors would like to thank S. Aarseth, D. Heggie, R. Spurzem, T. Lippert, K. Schilling, and W. Schroers for fruitful help and discussion. NSF grant AST 00-71099, NASA grants NAG 5-7019, NAG 5-6037, and NAG 5-9046. Technical help and computer resources are provided by $N I C$ in Jülich, $H L R S$ in Stuttgart, $T R A C S$ and $E P C C$ in Edinburgh, $Z I B$ in Berlin, $S S C$ in Karlsruhe, University of Heidelberg, Rutgers University, and University of Kiel, and by the San Diego Supercomputer Center.

The software will be available soon via:

http://www.physics.rutgers. edu/ marchems/ 